Thorax (1972), 27, 219.

\title{
Correction of type IV (mixed level) total anomalous pulmonary venous drainage
}

\author{
M. C. NELIGAN, E. O'MALLEY, and S. BLAKE \\ Departments of Cardio-thoracic Surgery and Cardiology, The Mater Misericordiae Hospital, Dublin
}

Successful correction of type IV (mixed level) total anomalous pulmonary venous connexion (TAPVC) in a 5-year-old boy is reported. The actual pathway of the pulmonary venous drainage in this case has not previously been described in the literature.

The lungs arise from a division of the foregut. Initially, the venous drainage from the anlage of the lung is to the systemic venous system, that is, the superior vena cava, innominate veins, coronary sinus, ductus venosus, and inferior vena cava. The pulmonary venous plexus then connects with the left atrial portion of the heart, usually by a single stem which is progressively absorbed into the posterior wall of the left atrium to form the four definitive pulmonary veins. If this junction fails to take place, one or other of the forms of anomalous pulmonary venous drainage may occur. The classification of TAPVC proposed by Darling, Rothney, and Craig (1957) is that generally employed. These authors divided cases on the basis of the anatomic location of the pulmonary-systemic venous junction. Those cases in which the drainage was to the superior vena cava or its branches were designated as type $I$ or supracardiac. Cases in which the pulmonary veins drained directly into the right atrium or through an enlarged coronary sinus were labelled as type II or cardiac. Type III drainage, or the infracardiac variety, comprised cases in which the pulmonary veins connected to the inferior vena cava or portal system. Type IV or mixed level drainage was simply a combination of types I to III as described above.

An atrial septal defect is present in nearly all cases. In its absence or if it is insignificant in size, the chance of survival beyond infancy is poor. Another factor against the possibility of successful surgical correction is the development of pulmonary hypertension. This may develop as the result of a large left-to-right shunt, or an increased pulmonary vascular resistance, or owing to the obstruction of pulmonary venous return from external compression. This latter variety is most often seen in the infracardiac form of the condition.

\section{CASE REPORT}

M.W., a 5-year-old boy, presented at this hospital in May 1970 with a history of recurrent respiratory infections. His parents had also noted that he tired easily on exertion and could not keep up with his companions. On examination he was below the normal parameters for both height and weight. His pulse rate was 82 per minute, sinus rhythm. His jugular venous pressure was raised $2 \mathrm{~cm}$ of water. A right parasternal impulse was both seen and felt. A soft systolic murmur was heard widely over the praecordium. This was best appreciated in the third left intercostal space. The second sound in the pulmonary area was widely split and fixed.

The remainder of the physical examination was uninformative. The haemoglobin was $12.3 \mathrm{~g}(84 \%)$. Other routine laboratory investigations were unremarkable. The electrocardiogram showed right axis deviation and right ventricular hypertrophy. The initial chest radiograph (Fig. 1) showed moderate cardiac enlargement with increased pulmonary vascularity. This increase in pulmonary vascular markings was more marked on the right side. However, the specific radiological characteristics of the 'scimitar' syndrome described by Neill, Ferencz, Sabiston, and Sheldon (1960), were not in evidence. The superior mediastinum was widened, giving rise to the 'figure of eight' or 'snowman' appearance.

Selective angiography was performed on two occasions in an endeavour to delineate the precise anatomy of the pulmonary veins. From the radiographs it was apparent that the left lung drained into an ascending vertical vein which joined the left innominate vein (Fig. 2). The pathway from the right lung was rather obscure; a common vein from the hilum divided at the right border of the heart. One branch appeared to pass beneath the diaphragm, its termination not being apparent. The other branch passed medially behind the oblique sinus of the pericardium, appearing to end in a large tortuous variscosity (Fig. 3).

Right-sided cardiac catheterization was undertaken immediately prior to one of these angiograms. The 


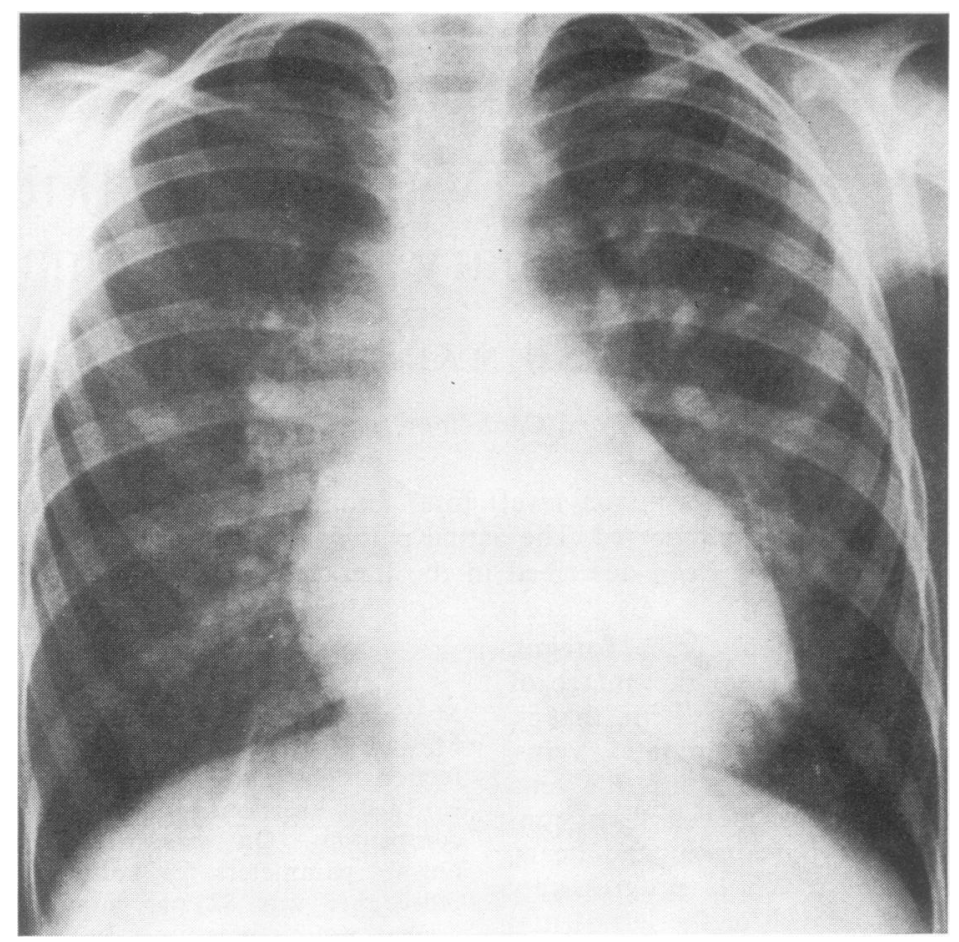

FIG. 1. Initial chest radiograph showing increased vascularity and 'snowman' appearance.

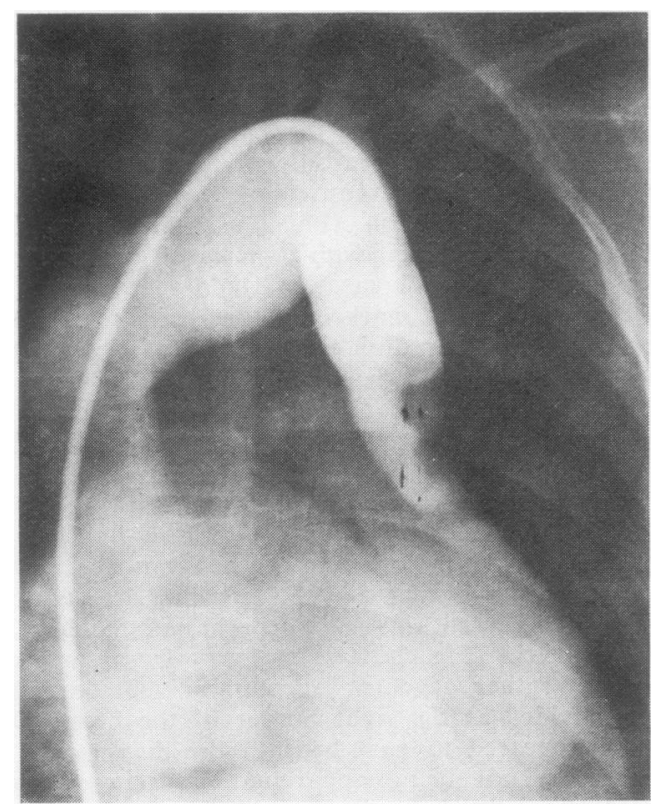

FIG. 2. Selective angiogram with catheter in ascending vertical vein which joins left innominate vein; entry of left pulmonary veins can be seen.
FIG. 3. Selective angiogram with catheter in rig pulmonary artery. Both branches of the right pulmonary vein are visualized. One passes below the diaphragm, and one ends in a tortuous varicosity, which forms the lon irregular opacity behind the left ventricle. 
findings were those of a marked oxygen step-up in the superior vena caval samples, to 14.2 vol $\%$ from 8.2 vol $\%$ in the right subclavian vein. $\mathbf{A}$ high inferior vena caval specimen had an oxygen content of $11.5 \mathrm{vol} \%$, possibly indicating a lesser degree of step-up at this site. The oxygen capacity of the blood was 15.9 vol \% and the pulmonary arterial and systemic arterial oxygen saturations were identical at $89 \%$.

OPERATIVE FINDINGS At operation the heart was exposed through a median sternotomy and vertical pericardiotomy. Both the superior and inferior venae cavae were noted to be unusually large. The right atrium and the right ventricle were also enlarged, the left side of the heart appearing small.

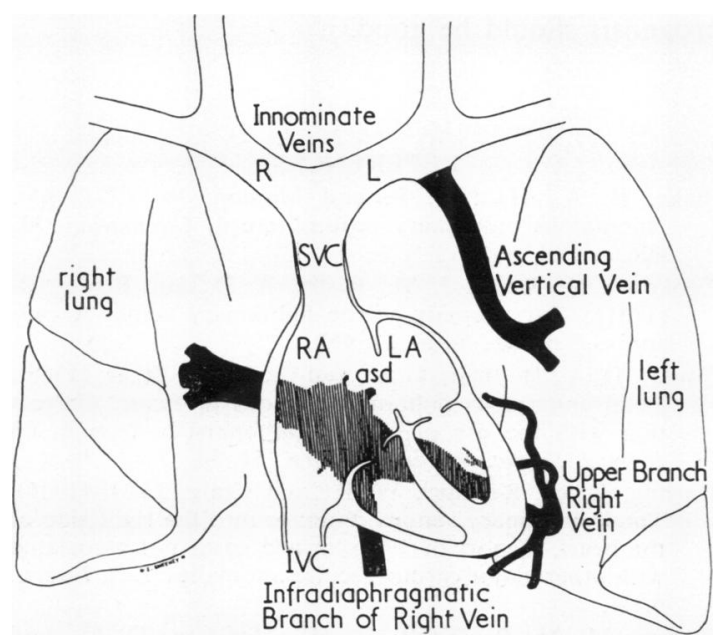

FIG. 4. Diagrammatic representation of the situation found at operation.

The tortuous dilatation of the upper branch of the right pulmonary vein has been omitted for clarity. Its distal branches did not communicate with the veins of the left lung.

It was necessary to open both pleurae to ascertain the exact anatomy of the pulmonary venous drainage. Both lungs appeared grossly normal and the pattern of the pulmonary venous drainage was as depicted (Fig. 4). On the right side a common vein from the hilum of the lung divided in two behind the heart. The inferior of these two branches passed through the diaphragm. Its termination was not seen. The upper branch passing behind the oblique sinus of the pericardium became a tortuous aneurysmal vessel. This broke up into a number of smaller veins which were thought to drain into radicles of the azygos and hemiazygos systems. No communication with the veins of the left lung was demonstrated. The veins from the left lung joined to form an ascending vertical vein which was followed to its junction with the left innominate vein. A moderate-sized atrial septal defect was palpated through the right atrial wall.

Cardiopulmonary bypass was begun using a RyggKyvsgaard disposable bubble oxygenator. Venous cannulae were passed through the right atrium into the superior and inferior venae cavae. The arterial return was through a cannula placed in the ascending aorta. A right ventricular venting system was em. ployed, the catheter being inserted through the infundibulum of the right ventricle and manipulated through the pulmonary valve. A side hole in the catheter below the pulmonary valve decompressed the right ventricle. The patient was cooled to $30^{\circ} \mathrm{C}$.

With the heart elevated an anastomosis was constructed between the back of the left atrium and the dilated upper branch of the right pulmonary vein. Tapes controlled the distal portion of this vein and also the inferior branch of the right vein. The anastomosis was $3 \mathrm{~cm}$ in length. Next a side-to-side anastomosis was constructed between the ascending vertical vein and the left atrial appendage. This anastomosis was approximately $2.5 \mathrm{~cm}$ in length. The systemic connexions of the pulmonary veins were then doubly ligated. Finally the right atrium was opened and the ostium secundum type atrial septal defect was closed. The initial anatomy was as depicted (Fig. 4) and the ultimate result was as shown (Fig. 5).

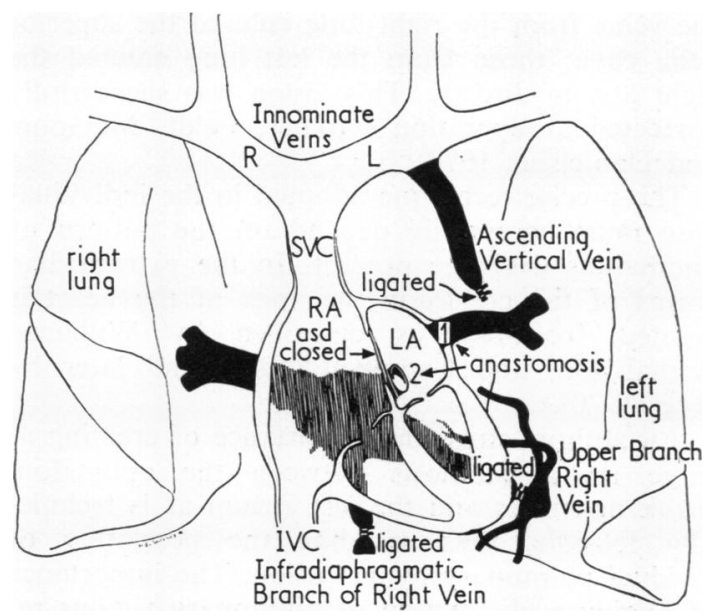

FIG. 5. Diagrammatic representation of the repair undertaken.

Cardiopulmonary bypass was discontinued after 100 minutes, the heart taking over well. The left atrial pressure at this stage was $20 \mathrm{~cm}$ of water and was similar in both the right and left pulmonary veins, thus indicating no stenosis at the sites of anastomosis. At this stage the arterial oxygen saturation was $99 \%$ and the mixed venous saturation $75 \%$.

The postoperative course was uneventful and the patient was discharged well. 


\section{DISCUSSION}

Most patients with TAPVC develop refractory cardiac failure early in life. In a collected series of 80 cases, Darling et al. (1957) found that $85 \%$ of these patients died under 2 years of age. Cooley, Hallman, and Leachman (1966) found that patients who survived longer than this period were good subjects for total surgical correction. The same authors also found that a systemic arterial saturation of greater than $85 \%$ in these patients was of good prognostic significance.

The spectrum of anomalous drainage presented in these cases is wide. Blake, Hall, and Manion (1965), reporting on 113 cases of the condition, described 27 variations encountered. None of these was similar to the pattern observed in this case. In the series described by Darling et al. (1957) there was only one case of type IV drainage. It was not similar to that described here. In a series of 62 patients submitted to surgical correction Cooley et al. (1966) had four patients with mixed level drainage. None of these survived the operative procedure. A series of 59 patients operated upon at the Mayo Clinic included only one patient with type IV drainage. In this case the veins from the right lung entered the superior vena cava; those from the left lung entered the right atrium directly. This lesion was successfully corrected at operation (Gomes, Feldt, McGoon, and Danielson, 1970).

The precise technique adopted in the individual case must necessarily depend on the pattern of anomalous drainage present. In the extracardiac forms of the condition the apex of the heart is rotated forwards, as described by Williams, Richardson, and Campbell (1964) and later by Roe (1970).

All authors stress the importance of creating as large an anastomosis between the anomalous trunk or trunks and the left atrium as is technically feasible, thus avoiding the possibility of residual pulmonary hypertension. The importance of avoiding obstruction of pulmonary venous return during the operation is also stressed by Wooler and Aberdeen (1968). The insertion of a venting system through the infundibulum of the right ventricle was useful in this respect.

In our patient the presence of the aneurysmal varicosity of the right pulmonary venous system allowed the construction of a satisfactory anastomosis between it and the back of the left atrium. Such pulmonary vein varicosities have been de- $\frac{\bar{\sigma}}{\bar{\rho}}$ scribed previously by Cooke, Evans, Kistin, and $\frac{\pi}{7}$ Blades (1951) and by Gottesman and Weinstein $\triangle$ (1959). The left atrial appendage in this child was moderate in size and its proximity to the ascending $\vec{\circ}$ vertical vein suggested its utilization for the second anastomosis. Although left atrial pressures $\vec{\omega}$ were high $\left(20 \mathrm{~cm} \mathrm{H}_{2} \mathrm{O}\right)$ at the end of the opera- $\mathrm{O}$ tion, there was no gradient across the sites of $\overrightarrow{\vec{x}}$ anastomosis. This suggests that left ventricular performance was the responsible factor for the $i v$ rather elevated left atrial pressure.

As the physiological abnormality in this condi- $\overrightarrow{0}$ tion is capable of complete correction the ultimate $\frac{\text { ? }}{3}$ prognosis should be good.

\section{REFERENCES}

Blake, H. A., Hall, R. J., and Manion, W. C. (1965). N Anomalous pulmonary venous return, Circulation, 32, 406.

Cooke, F. N., Evans, J. M., Kistin, A. D., and Blades, B. (1951). An anomaly of the pulmonary veins: A case study. J. thorac. Surg., 21, 452.

Cooley, D. A., Hallman, G. L., and Leachman, R. D. (1966). 을 Total anomalous pulmonary venous drainage. Correc- $\varrho$ tion with the use of cardio-pulmonary by-pass in $62 \overrightarrow{\overline{0}}$ cases. J. thorac. cardiovasc. Surg., 51, 88.

Darling, R. C., Rothney, W. B., and Craig, J. M. (1957). Total pulmonary venous drainage into the right side of the heart. Report of 17 autopsied cases not associated with other major cardiovascular anomalies. Lab. Invest., 6, 44.

Gomes, M. M. R., Feldt, R. H., McGoon, D. C., and Danielson, G. K. (1970). Total anomalous pulmonaryo venous correction: Surgical considerations and results 3 of operation. J. thorac. cardiovasc. Surg., 60, 116.

Gottesman, L., and Weinstein, A. (1959). Varicosities of the pulmonary veins: a case report and survey of the literature. Dis. Chest, 35, 322.

Neill, C. A., Ferencz, C., Sabiston, D. C., and Sheldon, H. (1960). The familial occurrence of hypoplastic righto lung with systemic arterial supply and venous drainage 'Scimitar syndrome'. Bull. Johns Hopk. Hosp., 107, 1. N

Roe, B. B. (1970). Posterior approach to correction of totar anomalous pulmonary venous return: Further experi- 0 ence. J. thorac. cardiovasc. Surg., 59, 748.

Williams, G. R., Richardson, W. R., and Campbell, G. S.o (1964). Repair of total anomalous pulmonary venous drainage in infancy. J. thorac. cardiovasc. Surg., 47, 199읃

Wooler, G. H., and Aberdeen, E. (1968). Modern Trends in Cardiac Surgery, 2nd ed. Butterworths, London. 